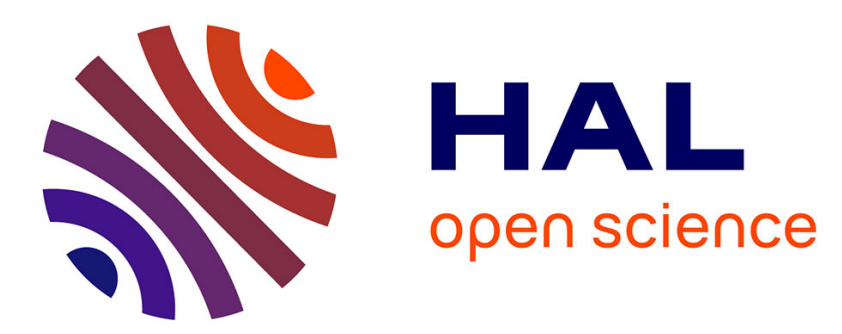

\title{
Randomized flow model and centrality measure for electrical power transmission network analysis
}

\author{
Enrico Zio, Roberta Piccinelli
}

\section{To cite this version:}

Enrico Zio, Roberta Piccinelli. Randomized flow model and centrality measure for electrical power transmission network analysis. Reliability Engineering and System Safety, 2010, 95 (4), pp.379-385. 10.1016/j.ress.2009.11.008 . hal-00609184

\section{HAL Id: hal-00609184}

https://hal-centralesupelec.archives-ouvertes.fr/hal-00609184

Submitted on 1 Oct 2012

HAL is a multi-disciplinary open access archive for the deposit and dissemination of scientific research documents, whether they are published or not. The documents may come from teaching and research institutions in France or abroad, or from public or private research centers.
L'archive ouverte pluridisciplinaire HAL, est destinée au dépôt et à la diffusion de documents scientifiques de niveau recherche, publiés ou non, émanant des établissements d'enseignement et de recherche français ou étrangers, des laboratoires publics ou privés. 


\title{
Randomized flow model and centrality measure for electrical power transmission network analysis
}

\author{
Enrico Zio, Roberta Piccinelli \\ Politecnico di Milano, Dipartimento di Energia, Via Ponzio 34/3, I-20133 Milano, Italy
}

\begin{abstract}
Commonly used centrality measures identify the most important elements in networks of components, based on the assumption that flow occurs in the network only along the shortest paths. This is not so in real networks, where different operational rules drive the flow. For this reason, a different model of flow in a network is here considered: rather than along shortest paths only, it is assumed that contributions come essentially from all paths between nodes, as simulated by random walks. Centrality measures can then be coherently defined. An example of application to an electrical power transmission system is presented.
\end{abstract}

\section{Acknowledgements}

This work has been partially funded by the Foundation pour une Culture de Securite Industrielle of Toulouse, France, under the research contract AO2006-01.

\section{Introduction}

Modern society is witnessing a continuous growth in the complexity of the infrastructure networks which it relies upon. Reliable electric power supply, for example, is crucial for many of the services that are taken for granted today; disturbances in the power supply have the potential of severely disrupting these indispensable services. This raises significant concern about reliability and resilience to disturbances and failures of various types of infrastructure systems, and a corresponding demand for methods capable of analyzing the vulnerabilities of these systems [1].

The developments contained in this paper are motivated by the interest in the analysis of electric power networks. In this context, the network analysis paradigm set up to study the dynamics of the relations in social networks has been previously utilized to analyze the vulnerability of electric power infrastructure systems [2, 3]. The focus of these types of studies is typically on analyzing the structural properties of the system from a topological point of view, i.e., considering only the connectivity properties of the network and not the actual physical flow through it $[4,5]$. Three drawbacks associated with the related measures of network performance are that they are based on:

- binary links among network nodes (or components), thus neglecting the strength of the connections (or links or arcs or edges); this has been pointed at as a limitation both in social networks, where the strength and depth of interpersonal relationships is of relevance $[6,7,8]$ and in engineered network infrastructures, where the capacities of the arcs connecting the components limit the flow among them [5]; 
- a simplified modeling scheme which assumes that flow (communication, in the social case) between a pair of components (persons, in the social case) in the network takes place only along the shortest path linking them $[8,9]$; this has been considered a limitation in many cases, because the flow from one node of a network to another is typically a global phenomenon which does not depend only on the links on the direct and shortest paths, since it is quite possible that information will take a more circuitous route; this is true both in social networks, where information may travel by random communication or be intentionally channeled through intermediaries, and in network infrastructures, where flow is channeled through selected routes, following the specific operative rules and constraints which apply to the system;

- a simplified modeling scheme which neglects the possibility of failures in the interconnections between pairs of linked components; this is particularly relevant for the engineered infrastructure networks made of fallible hardware and software, operated by (unfortunately) not error-free human operators.

In synthesis, when looking at the safety, reliability and vulnerability characteristics of an infrastructure such as the electric power transmission network, one should take into account the capacities of the transmission elements and their probability of failure, and examine the different transmission routes available to the flow. This would entail undertaking a complex and detailed mechanistic modeling effort of the entire network system, which is in practice often unfeasible, both with respect to its development and its computation. For this reason, a framework of analysis has been proposed to integrate models at different levels of detail, in a problem-driven approach to solution; complementation of network analysis, for performing an initial screening of the vulnerabilities of a critical infrastructure with object-oriented modeling, to further deepen the vulnerability assessment of the screened scenarios has been investigated as a feasible way to proceed in such direction [10].

To improve the physical description of the network characteristics within a network analysis for preliminary screening, a model based on random walks is here introduced as an extension of the model in [8] giving proper consideration to the following facts:

- each link connecting two nodes is characterized by a transmission capacity which cannot be exceeded;

- the capacities of the network lines are assumed to stochastically vary, to account for the inherent uncertainties;

- not only the links on the direct and shortest paths are considered in the analysis of the transmission of flow; this is achieved by a randomization of the direction of the flow in output from a node; the randomization is driven by the capacities of the outgoing links, with the highest capacity links most probably channeling the flow;

- the network interconnecting links are assumed fallible, with given probabilities;

- source generation and load demands are assumed to vary stochastically, to account for the fluctuations inherent in the network behavior and operation.

From the analysis of the network characteristics and behavior, it is also important to gain an understanding of the role that the elements of the infrastructure network play in determining the flow through it, as this can be of great practical aid to network designers and operators in providing indications for network protection. From a topological viewpoint, various measures of the importance of a network node, can be introduced. These so-called centrality measures, take into account the different ways in which a node interacts/communicates with the rest of the network. Classical topological centrality measures are the degree centrality [11, 12], the closeness centrality $[12 ; 13 ; 14]$, the betweenness centrality [12] and the information centrality [15]. The major drawback of these measures is that to assess the node importance they rely only on topological information based on the three previously mentioned model simplifications. Then, based on the model proposed in this paper an extension of 
the betweenness centrality measure of [16] is computed, to more realistically capture the importance of the role played by the different components in determining the flow through the network.

An application of the proposed approach is illustrated with reference to a power transmission network system of literature [17].

The paper is organized as follows. In Section 2, a description of the random walk flow propagation model is provided. In Section 3, the topological concept of betweenness centrality measure is recalled and then extended to its randomized flow definition. The results obtained on a case study of literature are discussed in Section 4. Conclusions are drawn in Section 5.

\section{Randomized flow model of a power transmission network infrastructure}

The topological interconnection of a power transmission system can be modeled as a network consisting of $\mathrm{N}$ nodes (also called vertexes) and $\mathrm{K}$ edges (also called arcs or lines): the buses of the electric grid are represented as nodes interconnected by undirected edges representing the transmission lines; $\mathrm{N}_{\mathrm{S}}$ nodes are power sources (generators), $\mathrm{N}_{\mathrm{T}}$ nodes are targets (loads) and the rest are transmission nodes. The $\mathrm{N} \times \mathrm{N}$ adjacency matrix $\left\{\mathrm{a}_{\mathrm{ij}}\right\}$ defines the topological structure of the network, i.e., the pattern of connectivity among its nodes, with the matrix entry $a_{i j}$ being equal to 1 if there is an edge linking $i$ and $j$ and 0 otherwise; the entries on the diagonal elements, $\mathrm{a}_{\mathrm{ii}}$, are undefined and for convenience they are set equal to 0 .

The matrix $\left\{\mathrm{q}_{\mathrm{ij}}\right\}$ defines the probabilities of failure of the links.

The capacities of the links are assumed to vary stochastically, to account for the uncertainties inherent in their behavior and operation; then, to each capacity value $\mathrm{w}_{\mathrm{ij}}$ is associated a probability distribution $\pi\left(\mathrm{w}_{\mathrm{ij}}\right)$ of the possible values.

The underlying strategy to model the flow in the network is to choose a source node, follow at random one of the departing links to one of its neighbors, take this as the source and iterate this process until the required target is reached. The random choice of the arc to follow is based on the actual capacity of each arc outgoing from the node: higher capacity arcs have larger probability to be selected as flow carriers.

Accordingly, the algorithm to evaluate the service reliability performance characteristics of the network, and its related vulnerabilities, consists of three nested cycles of randomization; the steps are as follows:

1. Sample the fault configuration of the network on the basis of the failure probabilities of each element (node or arc) of the system.

2. Sample the production from the sources, the demand at the targets and the capacity of the arcs.

3. Build the discrete cumulative distribution function of the capacities of the arcs leaving the source node and sample the flow direction from it.

4. Develop the flow propagation cycle, for each source:

4.1 the random walk of flow follows the arc sampled on the basis of the actual capacities of the arcs departing from the successive nodes traversed by the flow;

4.2 if the flow goes into an isolated node with no departing connections, the cycle ends;

4.3 the flow between a pair of nodes is accounted once (repeated flows between the same pair of nodes are neglected);

4.4 once the flow arrives at a target node, the capacities of the incoming arcs are checked: if their sum is larger than the maximum capacity of the node, an overload is recorded;

4.5 if the flow does not reach the target, a new source of random walk is sampled. If no flow arrives at any of the targets, then a blackout is recorded. 


\section{Randomized betweenness centrality measure}

Determining the critical elements of large-scale network infrastructures is an important issue for the reliability and the protection of the network. From a topological point of view, a number of centrality indices have been introduced as measures of the importance of the nodes in a network [18]. These indices take into account the different ways in which a node interacts and communicates with the rest of the network and have proved of value in the analysis and understanding of the role played by the elements in the network.

A classical topological centrality measure is the betweenness centrality [12]. This measure is based on the idea that a node is central if it lies between many other nodes, in the sense that it is traversed by many of the shortest paths connecting pairs of nodes. The topological betweenness centrality $\mathrm{C}_{i}^{\mathrm{B}}$ of a given node $i$ in a network $\mathrm{G}(\mathrm{N}$, $\mathrm{K}$ ), where $\mathrm{N}$ is the number of nodes and $\mathrm{K}$ is the number of links connecting them, is quantitatively defined as:

$$
C_{i}^{B}=\frac{1}{(N-1)(N-2)} \sum_{j, k \in G, j \neq k \neq i} \frac{n_{j k}(i)}{n_{j k}}
$$

where $\mathrm{n}_{\mathrm{jk}}$ is the number of topological shortest paths between nodes $\mathrm{j}$ and $\mathrm{k}$, and $\mathrm{n}_{\mathrm{jk}}(i)$ is the number of topological shortest paths between nodes $\mathrm{j}$ and $\mathrm{k}$ which contain node $i . \mathrm{C}_{\mathrm{i}}^{\mathrm{B}}$ assumes values between 0 and 1 and reaches its maximum when node $i$ falls on all geodesics (paths of minimal length between pairs of nodes).

From the definition, betweenness centrality can be regarded as a measure of the influence a node has on the spread of the flow through the network, of the extent to which a node has control over the flow between other nodes. In a network in which flow is entirely or at least mostly distributed along geodesic paths, the betweenness of a vertex measures how much flow will pass through that particular vertex.

In most networks, however, flow does not occur only along geodesic paths; in some cases, flow may not follow the ideal route to get from one place to another, and "wander around" in a random-like fashion or as directed by the system operative rules and constraints. In most cases, a realistic betweenness measure should include nongeodesic paths in addition to geodesic ones [19].

To account for this issue, a betweenness centrality measure based on the concept of network flow has been suggested [8]. The edges of a network are considered as channels of communication linking pairs of nodes; the value of the connection of two nodes $i$ and $j$ determines the capacity $w_{i j}$ of the channel linking them, or the amount of information that can pass between them. Information is assumed to flow along these channels. If $\mathrm{f}_{\mathrm{ij}}$ is the amount of information passing on a channel linking node $i$ directly to node $j$ then $f_{i j} \leq w_{i j}$, i.e., the amount of information flowing along a channel that links directly connected vertices cannot exceed the capacity of that channel. What is relevant here is not just the direct flow between connected nodes, but the overall flow between pairs of nodes along all the paths that connect them: if a node $\mathrm{i}$ is chosen as an information source, or transmitter, and another node $\mathrm{j}$ as an information sink, or receiver, information from $\mathrm{i}$ may reach $\mathrm{j}$ along an edge linking $\mathrm{i}$ directly to $\mathrm{j}$ or along any and all indirect paths that begin at $\mathrm{i}$, pass through one or more intermediate nodes and end at $\mathrm{j}$.

Thus, the flow between two nodes is a global phenomenon: it depends, not just on the capacity of the channel linking two nodes directly, but on the capacities of all the channels on all the paths - both direct and indirect that connect the two.

Ford and Fulkerson [16] introduced a model to determine the maximum flow from any source $i$ to any sink j. Let $\mathrm{m}_{\mathrm{jk}}$ be the maximum flow from a node $\mathrm{i}$ to a node $\mathrm{k}$ and let $\mathrm{m}_{\mathrm{jk}}(\mathrm{i})$ be the maximum flow from $\mathrm{j}$ to $\mathrm{k}$ that passes through node $\mathrm{i}$; then, the flow betweenness centrality measure may be quantitatively defined as [8]: 


$$
F C_{i}^{B}=\frac{\sum_{j=1}^{N} \sum_{k=j}^{N} m_{i k}(i)}{\sum_{j=1}^{N} \sum_{k=j}^{N} m_{j k}}
$$

Flow betweenness is based on the idea of maximum flow; the flow betweenness of a node $\mathrm{i}$ is defined as the amount of flow through $\mathrm{i}$ when the maximum flow is transmitted from source (s) to target ( $\mathrm{t}$ ), averaged over all $\mathrm{s}$ and $t$. Each edge in a network can be thought of as a transmission line carrying a flow of current. In general, more than a single unit of current can be carried between $s$ and $t$ by making simultaneous use of several different paths through the network.

In practical terms, the flow betweenness measures the betweenness of nodes in a network in which a maximal amount of information is continuously pumped between all sources and targets. Necessarily that flow still needs to "know" the ideal route (or one of the ideal routes) from each source to each target in order to realize the maximum flow. This still seems an unrealistic definition, in that it is often the case that flow does not take any sort of ideal path from source to target. To model this, a new betweenness centrality measure has then been introduced, the random walk betweenness [20]. Roughly speaking, the random walk betweenness of a node $\mathrm{i}$ is equal to the number of times that a random walk starting at $\mathrm{s}$ and ending at $\mathrm{t}$ passes through $\mathrm{i}$ along the way, averaged over all $\mathrm{s}$ and $\mathrm{t}$. This measure is appropriate to a network in which information wanders about essentially at random until it finds its target, and it includes contributions from many paths that are not optimal in any sense. Let $\mathrm{I}_{\mathrm{i}}^{\text {st }}$ be the current flowing from $\mathrm{s}$ to $\mathrm{t}$, through node i. Quantitatively the random betweenness centrality measure is defined as:

$$
R W C_{i}^{B}=\frac{\sum_{i=1, s<t}^{N} I_{i}^{s t}}{\frac{1}{2} N(N-1)}
$$

This measure seems an intuitively reasonable one to describe the fact that current will flow along all paths from source to target, and nodes that lie on no path from source to target get a betweenness of zero.

In this paper, an attempt to evaluate random betweenness centrality measures is made considering the physical characteristics of the transmission network in terms of length, capacity and failure probability of each transmission line, and types of nodes.

\section{Application}

The artificial transmission network system IEEE 14 BUS [17] is taken as reference case study. The network represents a portion of the American Electric Power System and consist of 14 bus locations connected by 20 lines and transformers, as shown in Figure 1. The transmission lines operate at two different voltage levels, 132 $\mathrm{kV}$ and $230 \mathrm{kV}$. The system working at $230 \mathrm{kV}$ is represented in the upper half of Figure 1, with 230/132 kV tie stations at Buses 4, 5 and 7. The system is also provided with voltage corrective devices in correspondence of Buses 3, 6 and 8 (synchronous condensers). Buses 1 and 2 are the generating units. 


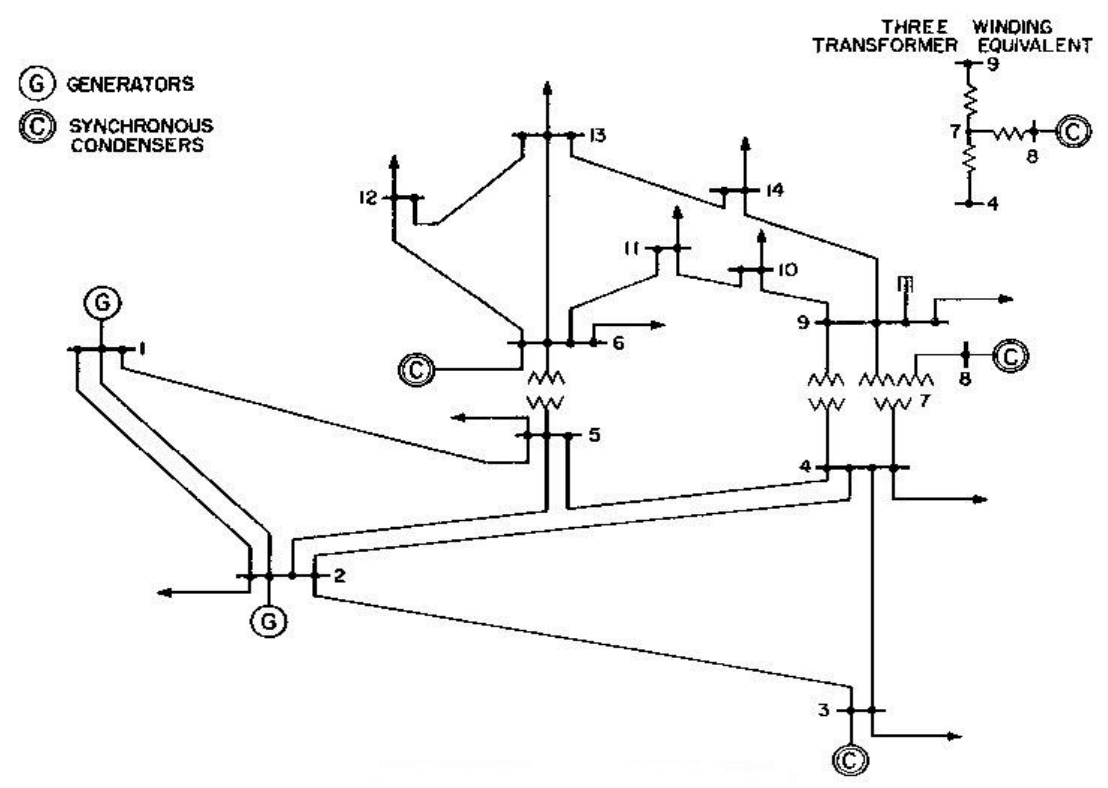

Figure 1. Transmission network IEEE 14 BUS [17].

To carry out the analysis, each network component is transposed into a node or edge of the representative topological network, as it is shown in Figure 2. Three different physical types of nodes are considered: source nodes (where the electricity is fed into the network), load nodes (where customers are connected) and transfer or transmission nodes (without customers or source).

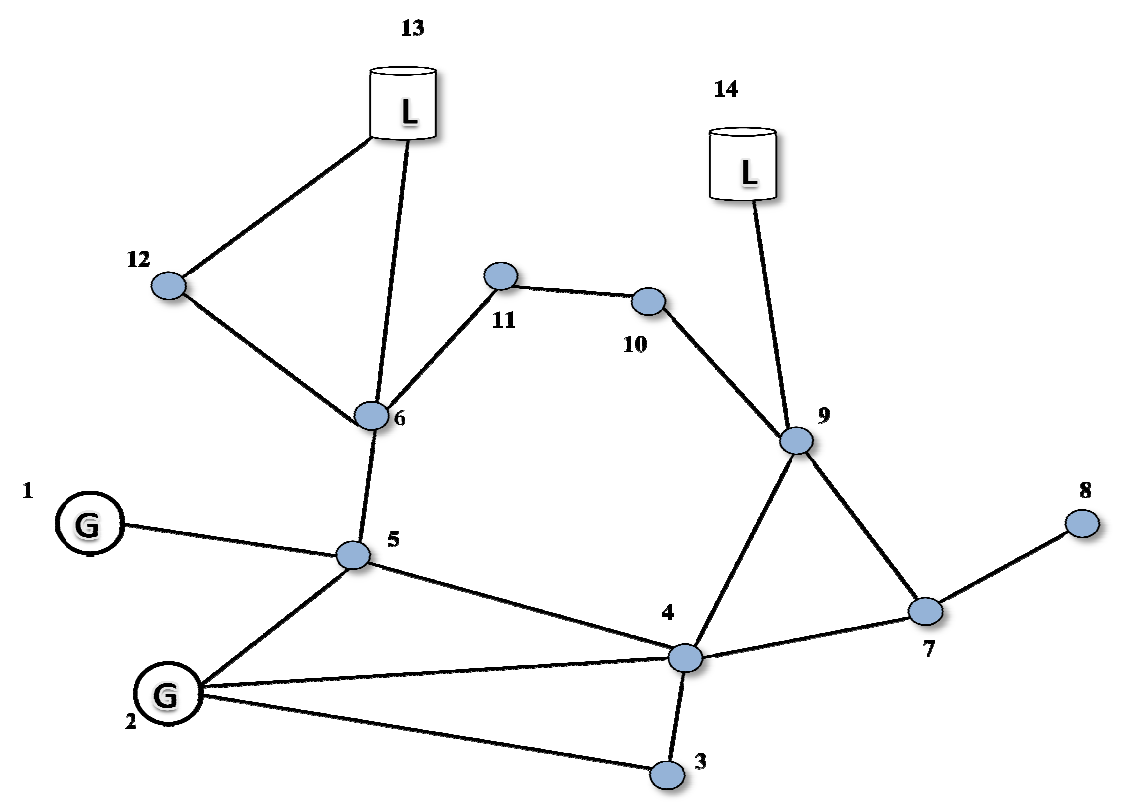

Figure 2. The IEEE 14 BUS transmission network's graph representation [21]. The white circles labeled with $\mathbf{G}$ represent the generator nodes, the colored circle nodes represent the transmission nodes and the white cylinders labeled with $\mathbf{L}$ represent the load nodes. 
Table I provides the adjacency matrix that defines the topological structure of the network.

$$
\left\{\mathrm{a}_{\mathrm{ij}}\right\}=\left|\begin{array}{cccccccccccccc}
0 & 1 & 0 & 0 & 1 & 0 & 0 & 0 & 0 & 0 & 0 & 0 & 0 & 0 \\
1 & 0 & 1 & 1 & 1 & 0 & 0 & 0 & 0 & 0 & 0 & 0 & 0 & 0 \\
0 & 1 & 0 & 1 & 0 & 0 & 0 & 0 & 0 & 0 & 0 & 0 & 0 & 0 \\
0 & 1 & 1 & 0 & 1 & 0 & 1 & 0 & 1 & 0 & 0 & 0 & 0 & 0 \\
1 & 1 & 0 & 1 & 0 & 1 & 0 & 0 & 0 & 0 & 0 & 0 & 0 & 0 \\
0 & 0 & 0 & 0 & 1 & 0 & 0 & 0 & 0 & 0 & 1 & 1 & 1 & 0 \\
0 & 0 & 0 & 1 & 0 & 0 & 0 & 1 & 1 & 0 & 0 & 0 & 0 & 0 \\
0 & 0 & 0 & 0 & 0 & 0 & 1 & 0 & 0 & 0 & 0 & 0 & 0 & 0 \\
0 & 0 & 0 & 1 & 0 & 0 & 1 & 0 & 0 & 1 & 0 & 0 & 0 & 1 \\
0 & 0 & 0 & 0 & 0 & 0 & 0 & 0 & 1 & 0 & 1 & 0 & 0 & 0 \\
0 & 0 & 0 & 0 & 0 & 1 & 0 & 0 & 0 & 1 & 0 & 0 & 0 & 0 \\
0 & 0 & 0 & 0 & 0 & 1 & 0 & 0 & 0 & 0 & 0 & 0 & 1 & 0 \\
0 & 0 & 0 & 0 & 0 & 1 & 0 & 0 & 0 & 0 & 0 & 1 & 0 & 1 \\
0 & 0 & 0 & 0 & 0 & 0 & 0 & 0 & 1 & 0 & 0 & 0 & 1 & 0
\end{array}\right|
$$

Table I. The IEEE 14 BUS adjacency matrix

Table II reports the failure rate values of the components of the transmission network, as inferred from literature data [22].

\begin{tabular}{ccccc}
\hline From BUS & To BUS & Failure rate/yr/100km & Failure rate/yr & Equipment \\
\hline 1 & 2 & 1.0858 & & $132 \mathrm{kV}$ transmission line \\
1 & 5 & 1.0858 & & $132 \mathrm{kV}$ transmission line \\
2 & 3 & 1.0858 & & $132 \mathrm{kV}$ transmission line \\
2 & 4 & 1.0858 & & $132 \mathrm{kV}$ transmission line \\
2 & 5 & 1.0858 & & $132 \mathrm{kV}$ transmission line \\
3 & 4 & 1.0858 & & $132 \mathrm{kV}$ transmission line \\
4 & 5 & 1.0858 & 0.01045 & $132 \mathrm{kV}$ transmission line \\
4 & 7 & & $132 / 230 \mathrm{kV}$ transformer \\
4 & 9 & 0.01045 & $132 / 230 \mathrm{kV}$ transformer \\
5 & 6 & 0.01045 & $132 / 230 \mathrm{kV}$ transformer \\
6 & 11 & 0.5429 & & $230 \mathrm{kV}$ transmission line \\
6 & 12 & 0.5429 & & $230 \mathrm{kV}$ transmission line \\
6 & 13 & 0.5429 & & $230 \mathrm{kV}$ transmission line \\
7 & 8 & & 0.01045 & $132 / 230 \mathrm{kV}$ transformer \\
7 & 9 & & 0.01045 & $132 / 230 \mathrm{kV}$ transformer \\
9 & 10 & 0.5429 & & $230 \mathrm{kV}$ transmission line \\
9 & 14 & 0.5429 & & $230 \mathrm{kV}$ transmission line \\
10 & 11 & 0.5429 & & $230 \mathrm{kV}$ transmission line \\
12 & 13 & 0.5429 & & $230 \mathrm{kV}$ transmission line \\
13 & 14 & 0.5429 & & $230 \mathrm{kV}$ transmission line \\
\hline
\end{tabular}

Table II. Failure data of the arcs of the IEEE 14 BUS transmission network

The failure probability of edge $\mathrm{ij}$ is defined as: 


$$
q_{i j}=1-e^{-\lambda_{i j} T}
$$

where $\lambda_{\mathrm{ij}}$ is the constant failure rate per unit time of the edge ij linking nodes $\mathrm{i}$ and $\mathrm{j}$ (column 4 in Table II) and $\mathrm{T}$ is the reference time for the analysis, here chosen equal to 1 year.

Because the failure rate data are usually given as function of the length of each transmission line (column 3 in Table II), in order to compute the failure probability (4.1) transmission line lengths must be known. In this work, these have been inferred from the available data as follows. The total impedance $Z_{\mathrm{ij}}$ of a transmission line ij is dependent on the length of the line $\mathrm{l}_{\mathrm{ij}}$ :

$$
Z_{i j}=\left(r_{i j}+j x_{i j}\right) l_{i j}
$$

where $\mathrm{r}_{\mathrm{ij}}$ is the resistance per unit length of arc $\mathrm{ij}$ and $\mathrm{x}_{\mathrm{ij}}$ is its reactance per unit length. While the resistance of the line depends both on the length and on the thickness of the wire, the reactance of the line depends only on the length [23]. Based on relation (4.2), the lengths of the transmission lines in the IEEE 14 BUS system have been obtained from literature data by taking a system power base of $100 \mathrm{MVA}$ and a conversion factor of $0.48 \Omega / \mathrm{km}$ [24]; lines containing transformers are considered to be zero-length lines (Table III).

\begin{tabular}{cccc}
\hline $\begin{array}{c}\text { From BUS } \\
\mathrm{i}\end{array}$ & $\begin{array}{c}\text { To BUS } \\
\mathrm{j}\end{array}$ & $\begin{array}{c}\text { Length }(\mathrm{km}) \\
\mathrm{l}_{\mathrm{ij}}\end{array}$ & $\begin{array}{c}\text { Failure Probability } \\
\mathrm{q}_{\mathrm{ij}}\end{array}$ \\
\hline 1 & 2 & 22 & 0.2125 \\
1 & 5 & 81 & 0.5768 \\
2 & 3 & 72 & 0.5338 \\
2 & 4 & 64 & 0.4932 \\
2 & 5 & 63 & 0.4884 \\
3 & 4 & 62 & 0.4828 \\
4 & 5 & 15 & 0.1498 \\
4 & 7 & - & 0.0104 \\
4 & 9 & - & 0.0104 \\
5 & 6 & - & 0.0104 \\
6 & 11 & 220 & 0.6970 \\
6 & 12 & 283 & 0.7847 \\
6 & 13 & 144 & 0.5425 \\
7 & 8 & - & 0.0104 \\
7 & 9 & - & 0.0104 \\
9 & 10 & 93 & 0.3978 \\
9 & 14 & 299 & 0.8027 \\
10 & 11 & 212 & 0.6843 \\
12 & 13 & 221 & 0.6999 \\
13 & 14 & 385 & 0.8762 \\
\hline
\end{tabular}

Table III. Length and failure probabilities of the transmission lines

The shortest lines are concentrated in the lower half of the network, which contains the generating units, while the longest lines belong to the upper half of the network, which contains the loads. The largest failure probabilities are concentrated on the edges directly connected with sources and with loads (edges $1-5,2-3,2$ -4 and $12-13,9-14,13-14)$. 
Source generation is sampled from a normal distribution with a mean value of 30 and a variance of 100 , in arbitrary units (a.u.). The values of the capacities of the network links are assumed all distributed according to a normal distribution of mean value 100 a.u. and a standard deviation of 10 a.u. The direction of flow is sampled on the actual capacities of the arcs. Once the flow arrives at a target node, the capacities of the incoming arcs are checked: if their sum is larger than the maximum capacity value of the node, an overload is recorded. The targets are absorbing nodes: the flow stops and the received flow is recorded for evaluating the network lost load and the network service efficiency; if no flow reaches any target, a service blackout is recorded.

The network performance characteristics computed on the basis of the above data are reported in Table IV:

\begin{tabular}{lc}
\hline Blackout (\%) & 0.82 \\
Overload (\%) & 0.00 \\
& \\
Network service efficiency & 0.18 \\
& \\
Network demanded load (a.u.) & 59.93 \\
Network received load (a.u.) & 10.92 \\
Network lost load (a.u.) & 49.01 \\
\hline
\end{tabular}

Table IV. Network performance indicators

where:

- blackouts and overloads are evaluated considering the average value of the flow that does not reach the targets or that exceeds the capacities of the transmission lines, respectively;

- the network demanded load is the average sum of the power generated from all the sources $s_{i}, i=1,2, \ldots$, $\mathrm{N}_{\mathrm{S}}$ :

$$
N D L=\sum_{i=1}^{N_{S}} s_{i}
$$

- the network received load is the average sum of the flow reaching the targets $t_{i}, i=1,2, \ldots, N_{T}$ :

$$
N R L=\sum_{i=1}^{N_{T}} t_{i}
$$

- the network lost load is obtained as the difference between demanded and received loads:

$$
N L L=N D L-N R L
$$

- the network service efficiency is obtained as the ratio between received and demanded loads:

$$
N S E=\frac{N R L}{N D L}
$$


In the artificial case considered, the network service efficiency is low and the blackout probability is high: very little of the generated power is received from the loads. This is caused by the high probability of failure of the transmission lines, due to their relatively high failure rates and large lengths.

To see the effect of the lines lengths, a second computation has been made using average transmission line lengths inferred from literature [24]. Two line lengths of 48 and $50 \mathrm{~km}$ have been considered (Table V).

\begin{tabular}{cccc}
\hline $\begin{array}{c}\text { From BUS } \\
\mathrm{i}\end{array}$ & $\begin{array}{c}\text { To BUS } \\
\mathrm{j}\end{array}$ & $\begin{array}{c}\text { Length }(\mathrm{km}) \\
\mathrm{l}_{\mathrm{ij}}\end{array}$ & $\begin{array}{c}\text { Failure probability } \\
\mathrm{q}_{\mathrm{ij}}\end{array}$ \\
\hline 1 & 2 & 48 & 0.4079 \\
1 & 5 & 48 & 0.4079 \\
2 & 3 & 48 & 0.4079 \\
2 & 4 & 48 & 0.4079 \\
2 & 5 & 48 & 0.4079 \\
3 & 4 & 48 & 0.4079 \\
4 & 5 & 48 & 0.4079 \\
4 & 7 & - & 0.0104 \\
4 & 9 & - & 0.0104 \\
5 & 6 & - & 0.0104 \\
6 & 11 & 50 & 0.2372 \\
6 & 12 & 50 & 0.2372 \\
6 & 13 & 50 & 0.2372 \\
7 & 8 & - & 0.0104 \\
7 & 9 & - & 0.0104 \\
9 & 10 & 50 & 0.2372 \\
9 & 14 & 50 & 0.2372 \\
10 & 11 & 50 & 0.2372 \\
12 & 13 & 50 & 0.2372 \\
13 & 14 & 50 & 0.2372 \\
\hline
\end{tabular}

Table V. Length and failure probabilities of the arcs

In this case, the lower half of the network has the largest failure probability. The network performance characteristics are reported in Table VI.

\begin{tabular}{lc}
\hline Blackout (\%) & 0.44 \\
Overload (\%) & $3.33 \cdot 10^{-4}$ \\
& \\
Network service efficiency & 0.60 \\
& \\
Network demanded load (a.u.) & 59.93 \\
Network received load (a.u) & 36.24 \\
Network lost load (a.u) & 23.70 \\
\hline
\end{tabular}

Table VI. Network performance indicators 
With respect to the previous case, targets now receive a larger load, the service efficiency is higher and the blackout probability is lower. This is due to the values of the transmission lines failure probabilities, which are smaller than in the previous case because of the smaller line lengths.

Finally, the centrality betweenness based on the proposed random walk model has been computed for the two examples. The results are shown in Figure 3.

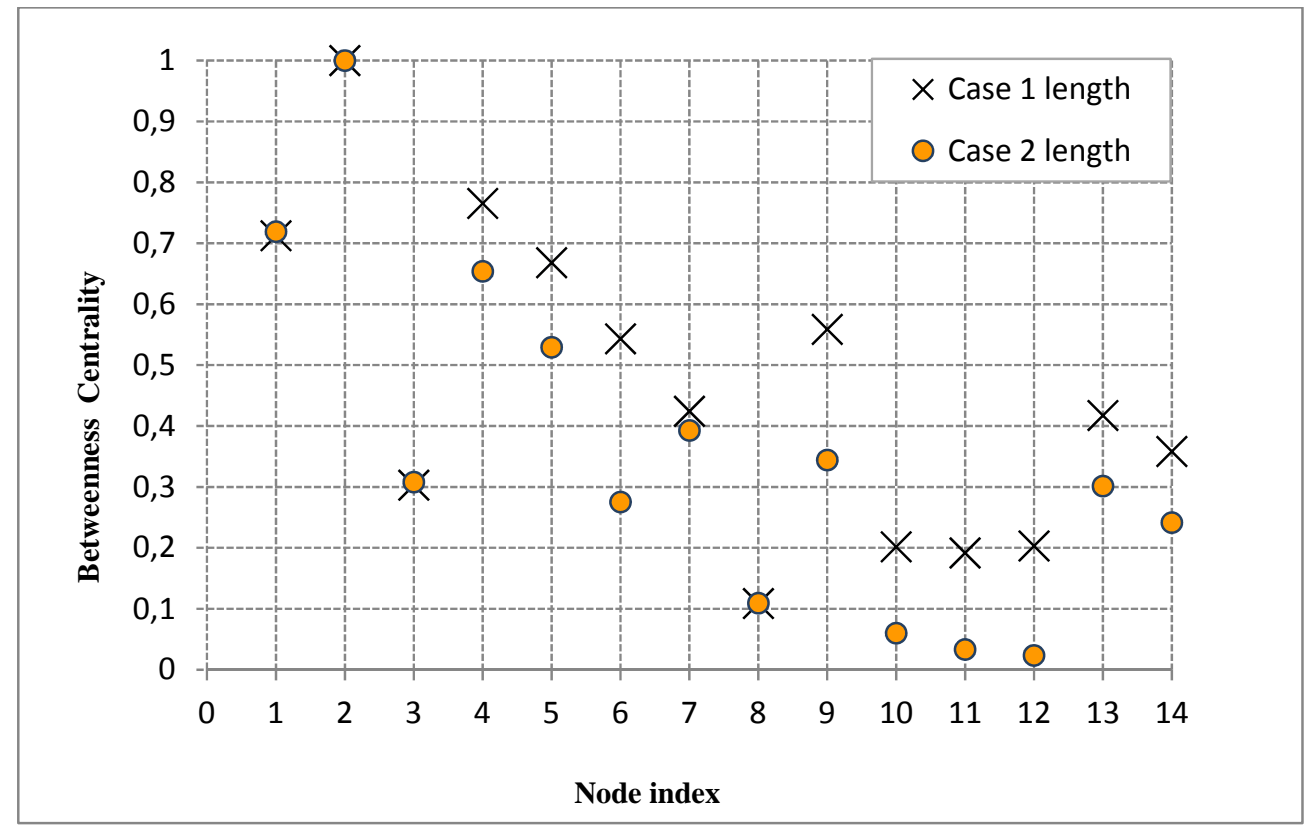

Figure 3. Betweenness centrality according to the random flow model proposed.

Betweenness values evaluated for case 1 ( $\times$, Figure 3 ) using higher lengths computed from eq. (4.2), are equal or higher than the values obtained for case 2 using smaller average lengths ( $\odot$, Figure 3 ), due to the associated larger failure probabilities. Equal values are obtained for nodes 1 and 2, i.e., the source nodes, for node 3 and for node 8 which is an isolated node (Figure 1). The lower half of the network (nodes 1, 2, 3, 4 and 5), which contains the generating units (nodes 1 and 2), has higher values of betweenness than the upper half, which contains the load nodes 13 and 14. Nodes 10,11 and 12 act as a tie for the flow. Node 2, which represents a source node and a transfer node as well, is the most important from the betweenness point of view, in both studies.

\section{Conclusions}

In previous works, network analysis has been shown suitable for a preliminary analysis of complex infrastructures aimed at identifying structural criticalities, e.g. the most connected nodes, shortest path lengths of connection, most vulnerable nodes, etc. Limitations of the analysis relate to the neglecting of the actual capacities of the links, their probabilities of failures and the fact that flow among network nodes is typically a global phenomenon, not restricted to only direct, shortest paths as typically assumed. 
To overcome some of these limitations, in this paper a model of random flow propagation has been introduced and the topological concept of betweenness centrality has been accordingly extended to account for the random flow propagation across the network. The randomization of the flow out of a node is driven by the capacity values of its outgoing links and allows non-geodesic paths to be travelled by the flow. Variability in the behavior and operation of the links, source and target nodes is also accounted for by varying stochastically the capacities, the productions and demands, respectively.

The modeling approach has been applied to the artificial transmission network system of the IEEE 14 BUS and indications derived from the betweenness centrality measure values have been analyzed for different transmission lines lengths. Each equipment of the system has been transposed into a node or edge of the representative network and the length of the arcs has been calculated for two cases: case 1, in which the lengths have been obtained from the impedances of the lines and case 2, in which average line lengths have been considered from literature data. The network performance characteristics and the random walk betweenness centrality measures have highlighted the weaknesses of the network structure, for the failure data used.

\section{Acknowledgments:}

The authors wish to thank Prof. Maurizio Delfanti and Dr.Mauro Pozzi of the Department of Energy of the Politecnico di Milano, for their contribution to the work.

\section{References}

[1] Albert, R., Albert, I., Nakarado, G.L., "Structural vulnerability of the North American power grid", Phys. Rev E 69, 025103 (R), (2004).

[2] Holmgren, A.J., "Using Graph Models to Analyze the Vulnerability of Electric Power Networks", Risk Analysis, Vol. 26, No. 4 (2006).

[3] Zio, E., Petrescu, C.A., Sansavini, G., "Vulnerability analysis of a power transmission network", Proceedings of PSAM9 - International Probabilistic Safety Assessment and Management Conference, Hong Kong, China, 18-23 May (2008).

[4] Cadini, F., Zio, E., Petrescu, C.A., "Using centrality measures to rank the importance of the components of a complex network infrastructure", CRITIS'08 - International Workshop on Critical Information Infrastructures Security, Frascati (Rome), Italy, 13-15 October (2008).

[5] Hines, P., Blumsack, S., "A Centrality Measure for Electrical Networks", Proceedings of the $41^{\text {st }}$ Hawaii International Conference on System Sciences (2008).

[6] Festinger, L., "The analysis of sociograms using matrix algebra", Human Relations 10, pp. 153 - 158 (1949).

[7] Yan, X., "A fuzzy set analysis of sociometric structure.” Journal of Mathematical Sociology 7 pp 159 - 180 (1988).

[8] Freeman, L.C., et al., "Centrality in valued graph: a measure of betweenness based on network flow", Social Networks 13, 141-154, (1991).

[9] Stephenson, K., Zelen, M., "Rethinking centrality: Methods and examples.", Social Networks 11, pp. 1 - 37 (1989).

[10] Eusgeld I., Kröger W., Sansavini G., Schläpfer M., Zio E., "The role of network theory and object-oriented modeling within a framework for the vulnerability analysis of critical infrastructures", Journal Reliability Engineering \& Systems Safety, Vol. 94, No 5, pp.954-963, 2009.

[11] Nieminen J., On Centrality in a Graph, Scandinavian Journal of Psychology, n.15, 1974. 
[12] Freeman, L.C., "Centrality in Social Networks: Conceptual Clarification”, Social Networks, 1, pp.215 239, (1979).

[13] Sabidussi G., The Centrality Index of a Graph, Psychometrika, n.31.1966.

[14] Wasserman S., Faust K., Social Networks Analysis, Cambridge U.P., Cambridge, UK.

[15] Latora V. and Marchiori M, A Measure of Centrality Based on the Network Efficiency, New Journal of Physics 9, 188, (2007).

[16] Ford, L.R. and Fulkerson, D.R.., "Maximal flow through a network", Canada Journal of Mathematics 8, 399 404, (1956).

[17] The IEEE 14 BUS data can be found on: http://www.ee.washington.edu/research/pstca/.

[18] Zio, E., "From Complexity Science to Reliability Efficiency: A new Way of Looking at Complex Network Systems and Critical Infrastructures", Int. J. Critical infrastructures, Vol. 3, Nos. 3/4, pp.488 - 508, (2007).

[19] Borgatti, S.P., "Centrality and Network Flow”, Social Networks 27, 55 -71, (2005).

[20] Newman, M.E., "A measure of betweenness centrality based on random walks", Preprint condmat/0309045, (2003).

[21] Pajek, Program for large network analysis : http://vlado.fmf.uni-lj.si/pub/network/pajek/.

[22] Billington, R., Li W., Reliability Assessment of electric power system using Monte Carlo Methods, pp 19 20, (1994).

[23] Grisby, L.L., Power Systems, CRC Press, (2007).

[24] IEEE Transaction on Power systems, Vol.14, No. 3, August 1999. 